\title{
High incidence of type II autoantibodies in pernicious anaemia
}

\author{
H M Waters, D W Dawson, J E Howarth, C G Geary
}

\begin{abstract}
Aims: To investigate the incidence of type II autoantibodies to intrinsic factor in pernicious anaemia.

Methods: Three hundred and forty four serum samples submitted for intrinsic factor antibody (IFAB) analysis on clinical or laboratory grounds were tested by an established radioassay and a new enzyme linked immunosorbent assay (ELISA) method for type I and total IFAB, respectively. Sixty of these were found to be positive by ELISA; this method was used to test further, 40 samples of adequate volume for types I and II antibodies.

Results: Type II antibodies were detected in 39 of the 40 sera tested. A comparative analysis indicated that seven samples contained pure type II antibody, being positive for total and type II by ELISA, but negative for type I by both the ELISA and radioassay technique.

Conclusions: The occurrence of type II antibody, both alone and in combination with type I, seems to be more common than has previously been recognised, and emphasises the advantage of using a technique which will detect both types of antibody.
\end{abstract}

(f Clin Pathol 1993;46:45-47)

The demonstration of circulating antibodies to intrinsic factor is virtually diagnostic of pernicious anaemia. ${ }^{1}$ Two types of intrinsic factor antibody (IFAB) are known to exist. ${ }^{2}$ Type I blocks the intrinsic factor-cobalamin binding site, preventing uptake of the vitamin. Type II reacts with an alternative antigen site on the intrinsic factor molecule and may prevent the attachment of the intrinsic factor-cobalamin complex to the ileal binding sites. ${ }^{3}$

Traditionally, methods of testing for IFABs have been based on functional methods for the detection of type I antibody. The reported prevalence of this type has ranged from $31 \%$ to $76 \% .^{34}$ The type II antibody has generally been regarded as being of lesser diagnostic importance, occurring less frequently and virtually always in association with type $I^{4}$ Demonstration of the type II variety is not carried out on a routine basis due, principally, to the cumbersome and insensitive nature of available methodology. Recently, immunoassay methods have been described which are capable of detecting both types of antibody, ${ }^{56}$ or type II alone. ${ }^{7}$

We report our findings regarding the inci- dence of type II antibodies using an ELISA method based on a two site solid phase principle. ${ }^{6}$

\section{Methods}

ELISA FOR TOTAL, TYPE I, AND TYPE II ANTIBODY Methods of preparation and the test protocols were as described previously, ${ }^{6}$ except that the solid phase support used in this study was the well of a polystyrene microtitre plate. ${ }^{8}$ For the detection of total antibody, human intrinsic factor, attached to the wells of a microtitre plate, first captures type I and type II IFABs, which are subsequently demonstrated by the attachment of an intrinsic factor/horseradish peroxidase conjugate and the colour produced by the addition of a suitable substrate. For the individual type I and type II antibody assays, the reactants are modified as follows. Addition of a $B_{12}$ intrinsic factor complex to the patient's serum absorbs out type II antibody and only type I reacts in the assay. Prior addition of $B_{12}$ to the intrinsic factor/horseradish peroxidase conjugate occupies the $B_{12}$ binding sites so that only type II antibody will be detected. Multiple estimates (24 each) of the background and negative antibody serum response were carried out using the total antibody ELISA protocol. The serum pool used to measure the negative antibody response was obtained from a panel of blood donors. The ELISA technique did not produce any false positive responses in our previous study. ${ }^{6}$

\section{RADIOASSAY FOR TYPE I ANTIBODY}

This was carried out using the method of Ardeman and Chanarin. ${ }^{39}$

Three hundred and forty four serum samples submitted for routine IFAB analysis on clinical or laboratory grounds were tested by the radioassay and ELISA methods for type I and total intrinsic factor antibodies, respectively. Sixty of these were found to be positive by ELISA, and further testing for types I and II antibodies was performed on the 40 samples of adequate volume.

Relevant clinical and laboratory details were obtained, where possible, on the patients included in the study, either directly from the case notes, or by means of a questionnaire for a number of requests originating from other hospitals. All sera tested were from patients who had been found to have low serum $B_{12}$ concentrations. Pernicious anaemia was diagnosed in 31 patients by conventional means the Schilling Test; IFAB by the technique of Ardeman and Chanarin ${ }^{39}$; and response to 
supplements of vitamin $B_{12}$. In nine patients a diagnosis of pernicious anaemia was probable, but no absorption tests had been performed and the ELISA IFAB positive result was required for an absolute diagnosis.

Table 1 Background and IFAB negative serum responses

\begin{tabular}{lll}
\hline & Background & Negative serum pool \\
\hline $\mathrm{n}=$ & 24 & 24 \\
Mean (A490) & 0.032 & 0.055 \\
SD (A490) & 0.002 & 0.003 \\
Mean + 3SD & 0.038 & 0.064 \\
\hline
\end{tabular}

Note: As the negative serum pool is expressing the response obtained in the complete absence of antibody, this was only measured using the total IFAB ELISA method.

Table 2 Results for IFAB ELISA (total, type I, and type II) and radioassay techniques

\begin{tabular}{|c|c|c|c|c|}
\hline \multirow{2}{*}{$\begin{array}{l}\text { Sample } \\
\text { number }\end{array}$} & \multicolumn{3}{|c|}{$E L I S A$} & \multirow[b]{2}{*}{ Radioassay } \\
\hline & Total & Type I & Type II & \\
\hline 1 & Pos & Neg & Pos & Neg \\
\hline 2 & Pos & Pos & Pos & Pos \\
\hline 3 & Pos & Pos & Pos & Pos \\
\hline 4 & Pos & Pos & Pos & Pos \\
\hline 5 & Pos & Pos & Pos & Pos \\
\hline 6 & Pos & Neg & Pos & Neg \\
\hline 7 & Pos & Pos & Pos & Pos \\
\hline 8 & Pos & Pos & Pos & Pos \\
\hline 9 & Pos & Pos & Pos & Pos \\
\hline 10 & Pos & Pos & Pos & Neg \\
\hline 11 & Pos & Pos & Pos & Pos \\
\hline 12 & Pos & Pos & Pos & Pos \\
\hline 13 & Pos & Pos & Pos & Pos \\
\hline 14 & Pos & Pos & Pos & Pos \\
\hline 15 & Pos & Pos & Pos & Neg \\
\hline 16 & Pos & Pos & Pos & Pos \\
\hline 17 & Pos & Pos & Pos & Pos \\
\hline 18 & Pos & Pos & Pos & Pos \\
\hline 19 & Pos & Pos & Pos & Neg \\
\hline 20 & Pos & Pos & Pos & Neg \\
\hline 21 & Pos & Pos & Pos & Pos \\
\hline 22 & Pos & Pos & Pos & $\mathrm{Neg}$ \\
\hline 23 & Pos & Pos & Pos & $\mathrm{Neg}$ \\
\hline 24 & Pos & Neg & Pos & Pos \\
\hline 25 & Pos & Pos & Pos & Pos \\
\hline 26 & Pos & Pos & Pos & Neg \\
\hline 27 & Pos & Pos & Pos & Pos \\
\hline 28 & Pos & Pos & Pos & Pos \\
\hline 29 & Pos & Pos & Pos & Pos \\
\hline 30 & Pos & Pos & Pos & Pos \\
\hline 31 & Pos & Pos & Pos & Neg \\
\hline 32 & Pos & Pos & Pos & Neg \\
\hline 33 & Pos & Neg & Pos & Neg \\
\hline 34 & Pos & Pos & Pos & Pos \\
\hline 35 & Pos & Neg & Pos & Neg \\
\hline 36 & Pos & Neg & Pos & Neg \\
\hline 37 & Pos & Pos & Pos & Pos \\
\hline 38 & Pos & Neg & Pos & $\mathrm{Neg}$ \\
\hline 39 & Pos & Neg & Neg & Neg \\
\hline 40 & Pos & Neg & Pos & Neg \\
\hline
\end{tabular}

Table 3 Comparison of results obtained by radioassay and ELISA techniques

\begin{tabular}{llccl}
\hline & & \multicolumn{2}{c}{ Radioassay } & \\
\cline { 3 - 5 } & & Positive & Negative & \\
\hline ELISA & Positive & 23 & 17 & $\mathrm{n}=40$ \\
Total & Negative & 0 & 0 & $\mathrm{n}=0$ \\
ELISA & Positive & $\mathrm{n}=23$ & $\mathrm{n}=17$ & $\mathrm{n}=31$ \\
type I & Negative & 1 & $\mathrm{n}$ & $\mathrm{n}=9$ \\
ELISA & Positive & $\mathrm{n}=23$ & $\mathrm{n}=17$ & $\mathrm{n}=39$ \\
type II & Negative & 0 & 16 & $\mathrm{n}=1$ \\
& & $\mathrm{n}=23$ & $\mathrm{n}=17$ & \\
\hline
\end{tabular}

Table 4 Comparison of results obtained by ELISA type I and ELISA type II techniques

\begin{tabular}{lllll}
\hline & & \multicolumn{3}{c}{ ELISA type I } \\
\cline { 2 - 5 } & & Positive & Negative & \\
\hline ELISA & Positive & 31 & 8 & $\mathrm{n}=39$ \\
type II & Negative & 0 & 1 & $\mathrm{n}=1$ \\
\hline
\end{tabular}

\section{Results}

The mean absorbances obtained for the background and negative antibody responses are shown in table 1 . A negative serum cut-off was established at the mean absorbance $+3 \mathrm{SD}$ level, providing a $99 \cdot 8 \%$ confidence limit for the maximum absorbance produced by an IFAB negative serum.

Table 2 shows the results obtained for the 40 test sera by both the ELISA and radioassay techniques. No false positive reactions resulting from increased serum $B_{12}$ concentrations were obtained by either method. The ELISA results were subdivided into total, type I, and type II.

A comparative analysis of the results is presented in tables 3 and 4 . Of the 40 sera containing total antibody, 31 were positive and nine were negative for type I by ELISA; only 23 were positive by radioassay. Type II antibody was present in 39 of the sera tested.

Linear regression was carried out to determine the relation between the IFAB radioassay (results expressed as reduction in normal $B_{12}$ binding capacity) and the three versions of ELISA (results expressed as absolute absorbance). This showed a good correlation for the type I assay $(r=0.584)$, which lessened progressively for the total $(r=0.469)$ and type II $(r=0.377)$ methods, respectively.

\section{Discussion}

The ELISA method detected circulating type II intrinsic factor autoantibodies in 39 of the 40 sera tested. Nine of the sera produced negative results for type I, and, overall, the apparent antibody concentration was weaker in the type I assay than in the type II. Comparison of the results produced by the ELISA and radioassay methods indicates that seven of these nine contained type II antibodies alone, being positive for total and type II by ELISA, but negative for type I by both methods. Of the other two sera negative for type I antibody one (number 24), from a new case of pernicious anaemia, was strongly positive for total and type II, but borderline negative and weakly positive by type I ELISA and radioassay, respectively. The last serum (number 39 ) in this group produced a weak positive result with the total ELISA technique, although negative with type I ELISA, type II ELISA, and radioassay. This sample probably contains types I and II antibodies at titres too low to produce individual positive results, but a positive total response resulted from the cumulative effect of both antibody types. Of the remaining sera, a further nine produced positive responses by ELISA for both types I and II antibodies, but were negative by radioassay. This probably reflects differences in sensitivity between the two methods. The radioassay used is an established technique, ${ }^{39}$ the sensitivity of which can be enhanced, but this requires prior treatment of the serum. ${ }^{10}$ There was complete agreement with the remaining 22 samples.

Basing the diagnosis of pernicious anaemia in nine patients on the ELISA IFAB is considered reasonable as the test is of high 
specificity. The ELISA technique may be susceptible to false negative responses, but due to the specificity of the intrinsic factor-IFAB complex and the principle underlying the test, false positive reactions are highly improbable. However, statistically, the choice of 3 SD above the mean of the negative serum response to distinguish between positive and negative results could, theoretically, lead to a false positive interpretation with 1 in 500 random sera.

Until recently, available methods for the detection of type II IFABs have been inappropriate to the delineation of their true incidence or relevance. Sourial used staphylococcal protein $A$ as a specific ligand to bind IgG molecules. ${ }^{7}$ In this way, type II IFAB could be demonstrated through the capture of an intrinsic factor $/{ }^{57} \mathrm{Co}-\mathrm{B}_{12}$ /antibody complex by the ligand coupled to Sepharose as a solid phase matrix. Samples from patients with pernicious anaemia who were positive for type I antibody were selected for this study, and of the 15 patients tested, only six were found to be positive for type II. ${ }^{7}$

Conn developed a radioassay method which uses ${ }^{125}$ I-labelled hog intrinsic factor and demonstrates the formation of an intrinsic factor-IFAB complex. ${ }^{5}$ By modifying the reagents involved, both types I and II antibodies can be detected. ${ }^{112} \mathrm{He}$ found low titres of type II antibody in all positive sera tested, and these were always in association with the type I variety. ${ }^{11}$ We are in broad agreement with Conn in that all but one of the samples tested in our study contained type II antibody. However, the responses produced by both antibodies differ in the two methods. Our ELISA produced a higher response for type II than type $I$, and in addition, seven sera contained pure type II antibody. As the principle governing the detection of type I and type II IFAB is essentially the same for both methods, it is unlikely that reagent manipulation differences alone could account for the discrepancies. These are more likely to be related either to the production of the label or methodology used for the separation of reacted from unreacted reagents.

The ELISA method uses human intrinsic factor as the source of antigen, ${ }^{6}$ which is then conjugated to horseradish peroxidase (HRP) by the periodate-aldehyde method of Nakane and Kawoia. ${ }^{13}$ This method of linking proteins uses the relatively high carbohydrate content (about 18\%) of the HRP as a coupling site. In this way conjugates of high specific activity, retaining up to $70 \%$ HRP activity, can be produced. The ${ }^{125} \mathrm{I}-\mathrm{hog}$ intrinsic factor label used in the Conn assay is produced by the chloramine-T oxidation method and is stable on storage for up to four weeks ${ }^{5}$ whereas the enzyme-labelled intrinsic factor can be stored and used for periods in excess of 12 months. ${ }^{6}$ Systematic differences between the two assays may be due to interspecies differences between the two types of intrinsic factor used, damage to the intrinsic factor molecule by the labelling process, or deterioration on storage.

The separative procedure used in the ELISA method comprises human intrinsic factor attached to the wells of polystyrene microtitre plates as solid phase, whereas the radioiodine assay is carried out in liquid phase with separation effected by polyethylene glycol precipitation of any immune complex formed. The use of a solid phase of the type employed by the ELISA technique may reduce the type I antibody response slightly by restricting access to the $B_{12}$ binding site of the solid phase or enzyme labelled intrinsic factor. ${ }^{6}$ However, nine sera tested in this study were positive for type I antibody by ELISA and negative by radioassay, indicating that although the chromogenic response is weaker, the ability to demonstrate antibody is more sensitive than that of the radioassay. This suggests reduced uptake of label, producing an attenuation of the signal, rather than a problem with uptake of antibody by the solid phase intrinsic factor.

Our study indicates that the occurrence of type II antibody, both alone and in combination with type $I$, is a more common feature than has previously been recognised. The application of a technique of high specificity capable of detecting both types of antibody as a cost effective screening test ${ }^{6}$ would therefore enhance the role of IFAB detection in the investigation of $B_{12}$ deficiency. For an increased number of patients this would obviate the requirement for $B_{12}$ absorption tests, currently required to reach a final diagnosis of pernicious anaemia. ${ }^{1}$

1 Lindenbaum J. Status of laboratory testing in the diagnosis of megaloblastic anaemia. Blood 1983;61:624-7.

2 Roitt IM, Doniach D, Shapland C. Intrinsic factor autoantibodies. Lancet 1964;ii:469-70.

3 Chanarin I. Pernicious anaemia-autoimmune aspects. In: The Megaloblastic anaemias. Oxford: Blackwell Scientific Publications, 1969:550-74.

4 Chanarin I. Pernicious anaemia-Clinical pathology. In: The Megaloblastic anaemias. Oxford: Blackwell Scientific Publications, 2nd edn. 1979:362-5.

5 Conn DA. Intrinsic factor antibody detection and quantitation. Med Lab Sci 1986;43:48-52.

6 Waters HM, Smith C, Howarth JE, Dawson DW, Delamore IW. New enzyme immunoassay for detecting total, type I, and type II intrinsic factor antibodies. I Clin Pathol 1989;42:307-12.

7 Sourial NA. Rapid protein A assay for intrinsic factor and its binding antibody. $\mathcal{F}$ Clin Pathol 1988;41:586-72.

8 Waters HM, Howarth JE, Shanks D, Geary CG. A high capacity enzyme immunoassay for the detection of total, type I, and type II intrinsic factor antibodies. Med Lab Sci
1989;46Suppl 1:S52.

9 Ardeman S, Chanarin I. Assay of gastric intrinsic factor in the diagnosis of Addisonian pernicious anaemia. $\mathrm{Br} \mathfrak{F}$ Haematol 1965;11:305-14.

10 Nimo RE, Carmel R. Increased sensitivity of detection of the blocking (type I) anti-intrinsic factor antibody. $\mathrm{Am} \mathrm{J}$ Clin Pathol 1987;88:729-33.

11 Conn DA. Detection of type I and type II antibodies to intrinsic factor. Med Lab Sci 1986;43:148-51.

12 Conn DA. Intrinsic factor antibodies in relation to disease. Med Lab Sci 1986;43:220-4.

13 Nakane PK, Kawoia A. Peroxidase-labelled antibody: a new method of conjugation. $f$ Histochem Cytochem 1974 22:1084-91. 